\title{
Ingredient of Biomass Packaging Material and Compare Study on Cushion Properties
}

\author{
Fangyi Li, ${ }^{1}$ Kaikai Guan, ${ }^{1}$ Peng Liu, ${ }^{1}$ Gang Li, ${ }^{1,2}$ and Jianfeng Li ${ }^{1}$ \\ ${ }^{1}$ School of Mechanical Engineering \& Key Laboratory of High Efficiency and Clean Mechanical Manufacture (Ministry of Education), \\ Shandong University, Jinan 250061, China \\ ${ }^{2}$ Shandong Kerui Holding Group Co., LTD, Dongying 257067, China
}

Correspondence should be addressed to Gang Li; sduligang@126.com

Received 23 March 2013; Revised 22 July 2013; Accepted 6 August 2013; Published 6 January 2014

Academic Editor: Shaojun Li

Copyright (C) 2014 Fangyi Li et al. This is an open access article distributed under the Creative Commons Attribution License, which permits unrestricted use, distribution, and reproduction in any medium, provided the original work is properly cited.

\begin{abstract}
In order to reduce the white pollution caused by nondegradable waste plastic packaging materials, the biomass cushion packaging material with straw fiber and starch as the main raw materials had been synthesized. The orthogonal experiment was used to study the impact of mass ratio of fiber to starch, content of plasticizer, active agent, and foaming agent on the compressive strength of cushion material. Infrared spectrometer and theory of water's bridge-connection were used to study the hydroxyl groups among the fiber and starch. The results were demonstrated as follows: the mass ratio of fiber to starch had the most significant impact on compressive strength. When the contents of the plasticizer, the foaming agent, and the active agent were, respectively, $12 \%, 0.1 \%$, and $0.3 \%$ and the mass ratio of fiber to starch was $2: 5$, the compressive strength was the best up to $0.94 \mathrm{MPa}$. Meanwhile, with the plasticizer content and the mass ratio of fiber to starch increasing, the cushioning coefficient of the material decreased first and then increased. Comparing the cushion and rebound performance of this material with others, the biomass cushion packaging material could be an ideal substitute of plastic packaging materials such as EPS and EPE.
\end{abstract}

\section{Introduction}

At present, the cushion packaging products widely used are mainly made from foamed plastic, corrugated cardboard, honeycomb paper, paper pulp molding, and so forth. As the plastic packaging waste is nonrecyclable and nondegradable, it has caused serious white pollution and heavy pressure on the environment. Though the corrugated cardboard, the honeycomb paper, and the paper pulp molding materials are biodegradable, their raw material is mainly wood, which consumes too much limited forest resources in China. And also, water pollution is caused in the manufacturing process, as well as higher manufacturing cost. Therefore, these kinds of material could not be ideal alternatives for plastic packaging materials.

The biomass cushion packaging products are prepared through a special process, using plant fiber (straw fiber, bagasse fiber, etc.) and starch as the main raw material and plasticizer and foaming agent as the additives.
This kind of material is generally regarded as eco-friendly because of its "green characters," such as wide sources of raw material, simple preparation process, low cost, and no environmental pollution in the full life cycle. Nowadays, this material has become a research hotpot all over the world.

In the early process of this new subject, considerable studies have been performed on developing biomass cushion packaging product with natural fibers and bio polymers such as starch, polylactide (PLA), and rubber latex. And the production technology was optimized according to the raw materials and product requirements $[1-4]$. Shey et al. $[5,6]$ carried out tensile strength and extension tests according to the products developed, such as starch-based degradable films and fiber-based pallets, and then analysed the microstructure using the scanning electron microscope. Sawpan et al. $[7,8]$ studied the characteristics of plant fiber cushion material and got the relationship between the structural advantages and practical application of the material through 
some application tests. Kunanopparat et al. [9, 10] plasticized the main raw material, starch, of the biomass cushion material and obtained the variation trend and variation mechanism of mechanical properties and degradation property of the material under different plasticizing conditions. Jia et al. [11] researched on foaming molding process of the biomass tableware and obtained a more mature process. Rosa et al. [12] modified the natural fiber in order to obtain its better compatibility with other raw materials and then improved the flexibility of the biomass products by foaming process. Besides, some researchers discussed the foaming dies and production lines of biomass degradable products $[13,14]$. As can be concluded from the literatures above, scholars mostly focus on ingredients, preparation, foaming molding process, and production lines of the biomass degradable materials, and usually only impact of single factor in the ingredient on cushion properties was analysed. This study lacking of crosswise contrast has caused many problems as follows. The interaction of raw materials results in confusion in practical production. Because of lacking deep theoretical analysis about the ingredient impact mechanism, the ingredient process is hard to optimize. Besides, the biomass property test analysis is too much simple and lacks general tests and comparison with the same kind of cushion material, which hinders the marketization process of the biomass cushion packaging material.

Based on the previous systematic research of ours such as degradation performance, mechanical properties and environment friendly in full life cycle of the biomass cushion packaging product [15-19], further research on ingredient and cushion properties is carried out. Through the new experiments, the ingredient is optimized and as a result, the functional performance of the material is better. In this paper, the orthogonal experiment method is used to study the impact of the mass ratio of fiber to starch, the content of plasticizer, the active agent, and the foaming agent on the compressive strength of the biomass cushion packaging material. The best ingredient is obtained. Besides, single factor experiment method is used to study the impact of the mass ratio of fiber to starch and the content of plasticizer on the cushion performance. In the end, cushion performance and rebound performance of this new material is compared with EPS and EPE.

\section{Material Processing and Experiment Methods}

\subsection{Raw Material and Experiment Facilities}

(1) Main Raw Materials. Plant fiber (straw fiber): 60 mesh; corn starch: average particle diameter about $70 \mathrm{~nm}$; ammonium bicarbonate, stearic acid, glycerol, ethylene glycol, and so forth.

Plant fiber acts as the main bearing carrier of force, just like a framework. Corn starch, the mass ratio of which is the highest, plays an important role in filling and bonding with fiber and other additives. Ammonium bicarbonate, as the foaming agent, is used to generate the bubble microporous structure. Glycerol and ethylene glycol, as the plasticizers, plasticize the starch in order to improve the adhesion property with plant fiber. Other raw materials are mainly used for better quality of product molding.

(2) Experiment Facilities. Hot-embossing machine with double column, thermoforming mold (custom made), HHS2 electronic constant temperature water bath (Shanghai Kang Road Equipment Co., Ltd.), mixer HWT201 (ZhangqiuJiaYue Machinery Factory), and XYD-15K carton compression testing machine (Jinan new era assaying Co., Ltd).

\subsection{Experiment Methods}

2.2.1. Production Process. As shown in Figure 1, the process is divided into four parts: fiber fibrillation, starch plasticizing, additives, and molding.

(1) Fibrillation treatment of predried fiber was carried out after fiber was soaked in $5 \%(\mathrm{w} / \mathrm{v})$ sodium hydroxide aqueous solution for 3 hours. Beat the fiber till the standard of devillicate and then the fiber must be rinsed clean and dried [20]. Fiber fibrillation was to expose more active hydroxyl groups so that the hydroxyl groups could combine more easily.

(2) Starch and water were mixed by the mass ratio of $1: 4$, and then the starch was gelatinized after adjusting the temperature of the thermostatic water bath. While churning, the compound plasticizer, prepared using glycerin and polyethylene glycol according to a given volume ratio, was added in. The starch could be plasticized efficiently. As a result, better compatibility between starch and fiber and better rheological property of the slurry were obtained.

(3) Put the fiber after beating fibrillation treatment and plasticized starch into the mixer. After 20 minutes churning, foaming agent, surfactant, and other additives were added in and mixed up until homogeneous.

(4) Set the upper mold temperature at $200^{\circ} \mathrm{C}$, lower mold at $205^{\circ} \mathrm{C}$, and pressure at $5 \mathrm{MPa}$. Put the homogeneous phase of a given weight into the lower mold for a $45 \mathrm{~s}$ baking. Maintain the pressure and temperature stably for a 30 s drying and then take out the products.

2.2.2. Mechanical Property Experiment. Cushion performance and rebound performance are the dominant two mechanical property indexes of packaging materials, which are choosing to inspect the bearing capacity at the impact of external load and the resilience in the case of multiple load impact. Experiments of compression performance and rebound performance were carried according to the national standard of China in this paper. Besides, cushion performance is the most important factor in using constraints and characterization of packaging materials. The optimization 


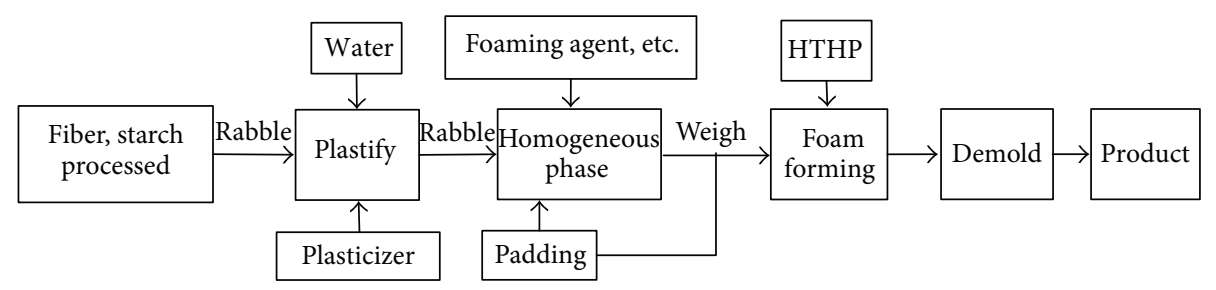

FIGURE 1: Biomass cushion packaging material forming processes.

TABLE 1: Experiment factors and levels.

\begin{tabular}{lcccc}
\hline \multirow{2}{*}{\begin{tabular}{c} 
Levels \\
\cline { 2 - 4 }
\end{tabular}} & \multicolumn{2}{c}{ Factors } \\
Mass ratio of fiber to starch & Content of plasticizer/\% & Content of active agent/\% & \multicolumn{2}{c}{ Content of foaming agent/\% } \\
A & B & 8 & 0.1 & 0.10 \\
\hline 1 & $2: 5$ & 10 & 0.3 & 0.12 \\
3 & $1: 4$ & 12 & 0.5 & 0.14 \\
\hline
\end{tabular}

objective of formula will focus on the compression performance mostly.

Compression Performance Experiment. Standard samples $(100 \times 100 \times 26 \mathrm{~mm})$ were prepared according to GB/T 81682008 China. According to the standard method A, made a pre-compaction of $5 \mathrm{~N}$ on the sample with the press plate of XYD-15K compression testing machine, measured the thickness of the sample as the original thickness, then loaded the press plate at a $12 \mathrm{~mm} / \mathrm{min}$ speed along the thickness direction of sample, recorded the compression force and the deformation accordingly.

The stress-strain curve of the sample was obtained through the test data, stress formula, and strain formula. The specific energy of the sample was equal to the area of the curve-side-triangle in the curve. As each curve-sidetriangle corresponded to a maximum stress, the cushion coefficient could be calculated. From the origin, draw different curve-side-triangles from small to large, and then obtain the functional relationship between the cushion coefficient and the maximum stress. The cushion coefficientmaximum stress curve $\left(\mathrm{C}-\sigma_{m}\right)$ of the sample was finally obtained.

Rebound Performance Experiment. According to GB/T 147451993 China, the standard samples were prepared. The XYD-15K compression testing machine was loaded at a $(12 \pm 3) \mathrm{mm} / \mathrm{min}$ speed along the thickness direction. When the deformation was about $50 \%$, maintain the pressure for 3 minutes. After unloading for $10 \mathrm{~s}$, use a vernier caliper to measure the thickness. Repeat the steps above for 3 times with the time interval of 1 minute. Calculate the rebound ratio with the following calculation formula:

$$
t_{j}=\frac{\left[T_{j}-\left(T_{i} / 2\right)\right]}{T_{i} / 2} \times 100 \% \ldots
$$

where $t_{j}$ is rebound ratio, $T_{i}$ is sample thickness before the compression, mm, $i=0,1,2 \ldots, T_{j}$ is sample thickness after the compression, $\mathrm{mm}$, and $j=1,2,3 \ldots$.

2.2.3. Infrared Spectrum Experiment. Grind $1 \mathrm{mg}$ sample and $150 \mathrm{mg} \mathrm{KBr}$ together into powder, and then transfer the powder into the compression die with the stainless shovel. Install the compression die on the cavity of tablet machine and apply a pressure of about $12 \sim 14 \mathrm{MPa}$ to make a transparent or translucent sheet. Take the compression die down and place it in the bracket of infrared spectrometer and set up the parameters. Operate the procedure to scan the sample while the wave number scanning range was $400-4000 \mathrm{~cm}^{-1}$. Print and save the spectra, and then analyze the peak-shaped ownership.

By the prophase single factor experiment and analyzing the formula and process of the biomass cushion packaging material, four factors, the mass ratio of fiber to starch, the content of plasticizer, the active agent, and the foaming agent, had the greatest impact on the cushion performance. So this experiment set three levels of each factor. Arrange four factors (A, B, C, D) and three levels L9 $\left(3^{4}\right)$ of orthogonal experiment to determine the optimum content of the main raw materials in the formula. Factor levels are shown in Table 1, orthogonal design and variance analysis were shown in Tables 2 and 3. During the experiment, the plasticizer was glycerol-ethylene glycol compound plasticizer prepared by the mass ratio of $1: 2$, the active agent was stearic acid, the foaming agent was ammonium bicarbonate, and the padding was stuffing calcium lignosulfonate with little impact on the cushion performance. The above additives have no harm to the environment, because of the full-degradability of them. 
TABLE 2: Experiment results.

\begin{tabular}{|c|c|c|c|c|c|}
\hline \multirow{2}{*}{$\begin{array}{l}\text { Experiment } \\
\text { numbers }\end{array}$} & \multicolumn{4}{|c|}{ Factors } & \multirow{2}{*}{ Compressive strength/MPa } \\
\hline & $\begin{array}{c}\text { Mass ratio of fiber to } \\
\text { starch }\end{array}$ & $\begin{array}{c}\text { Content of } \\
\text { plasticizer/\% }\end{array}$ & $\begin{array}{c}\text { Content of active } \\
\text { agent } / \%\end{array}$ & $\begin{array}{c}\text { Content of foaming } \\
\text { agent } / \%\end{array}$ & \\
\hline 1 & $1(2: 5)$ & $1(8)$ & $1(0.1)$ & $1(0.10)$ & 0.70 \\
\hline 2 & 1 & $2(10)$ & $2(0.3)$ & $2(0.12)$ & 0.90 \\
\hline 3 & 1 & $3(12)$ & $3(0.5)$ & $3(0.14)$ & 0.83 \\
\hline 4 & $2(1: 4)$ & 1 & 2 & 3 & 0.61 \\
\hline 5 & 2 & 2 & 3 & 1 & 0.81 \\
\hline 6 & 2 & 3 & 1 & 2 & 0.82 \\
\hline 7 & $3(1: 6)$ & 1 & 3 & 2 & 0.54 \\
\hline 8 & 3 & 2 & 1 & 3 & 0.61 \\
\hline 9 & 3 & 3 & 2 & 1 & 0.88 \\
\hline$x_{1}$ & 0.810 & 0.617 & 0.710 & 0.797 & \\
\hline$x_{2}$ & 0.747 & 0.773 & 0.797 & 0.753 & \\
\hline$x_{3}$ & 0.677 & 0.843 & 0.727 & 0.683 & \\
\hline Range $R$ & 0.133 & 0.226 & 0.087 & 0.114 & \\
\hline
\end{tabular}

Total mass of fiber and starch has the same ratio in each experiment.

TABLE 3: Significance analysis $(F)$ of orthogonal experiment.

\begin{tabular}{|c|c|c|c|c|c|c|}
\hline Variance sources & Quadratic sum/SS & DOF & Ratio of $F$ & $F_{0.10}$ & $F_{0.25}$ & Significance \\
\hline A & 0.027 & 2 & 0.766 & \multirow{4}{*}{3.110} & \multirow{4}{*}{0.384} & Less significant \\
\hline B & 0.081 & 2 & 2.298 & & & Less significant \\
\hline $\mathrm{C}$ & 0.013 & 2 & 0.369 & & & Little effect \\
\hline $\mathrm{D}$ & 0.020 & 2 & 0.567 & & & Less significant \\
\hline Error & 0.14 & 8 & & & & \\
\hline
\end{tabular}

\section{Results and Analysis}

3.1. Ratio Optimization. As shown in Table 2, the compressive strength of experiment 2, A1B2C2D2, was maximum. According to the value of ranges, the order of four factors' impact on compressive strength was $\mathrm{B}>\mathrm{A}>\mathrm{D}>\mathrm{C}$; that is, plasticizer $>$ the mass ratio of fiber to starch $>$ foaming agent $>$ active agent. The optimal horizontal combination was A1B3C2D1.

As was seen from Table 3, the difference of the impacts of four factors on the compressive strength was not remarkable. According to the $F$ value of each factor, the order of the impacts of the factors on the compressive strength was plasticizer $>$ the mass ratio of fiber to starch $>$ foaming agent $>$ active agent. The optimal horizontal combination was A1B3C2D1, consistent with the judgment of ranges. For absence of experiment A1B3C2D1 in Table 3, the verifying experiment had to be done. In this paper, three repeated experiments were done under the condition of combination A1B3C2D1, that is, the mass ratio of fiber to starch of $2: 5$, plasticizer content of $12 \%$, foaming agent content of $0.1 \%$, and active agent content of $0.3 \%$. The results showed that the compressive strength of the material could reach $0.94 \mathrm{MPa}$, which was better than the strength $0.90 \mathrm{MPa}$ in the orthogonal experiment of combination A1B2C2D2. Therefore, combination A1B3C2D1 was chosen as the optimum process condition.

\subsection{Cushion Performance}

3.2.1. Impact of Plasticizer on the Cushion Performance. The relationship between the cushion coefficients of different plasticizer contents of the material and the stresses is described in Figure 2. Experiment conditions were as follows: mass ratio of fiber to starch of $2: 5$, surfactant content of $0.3 \%$, and foaming agent content of $0.1 \%$.

As shown in Figure 2, under the same stress condition, different contents of plasticizer had a great influence on the cushion coefficient. When the plasticizer content was $10 \%$ or $12 \%$, the cushion coefficient was smaller, indicating better cushion performance. With the plasticizer content increasing, the cushion coefficient of the material decreased first and then increased. The main reasons were as follows. As the functional groups of the plasticizer damages the recrystallization of starch, the recrystallinity decreases, which leads to better thermoplasticity and hydrophilicity of starch. Then in the foaming molding process with hydrophilic plant fiber, better bound forms and microstructure of the biomass material is formed and hence a better microstructure. So, the flexibility of the material matrix increases and its cushion performance becomes better [10]. However, when the plasticizer content is excessive, the functional groups of the plasticizer will obstruct the normal molecular combination 


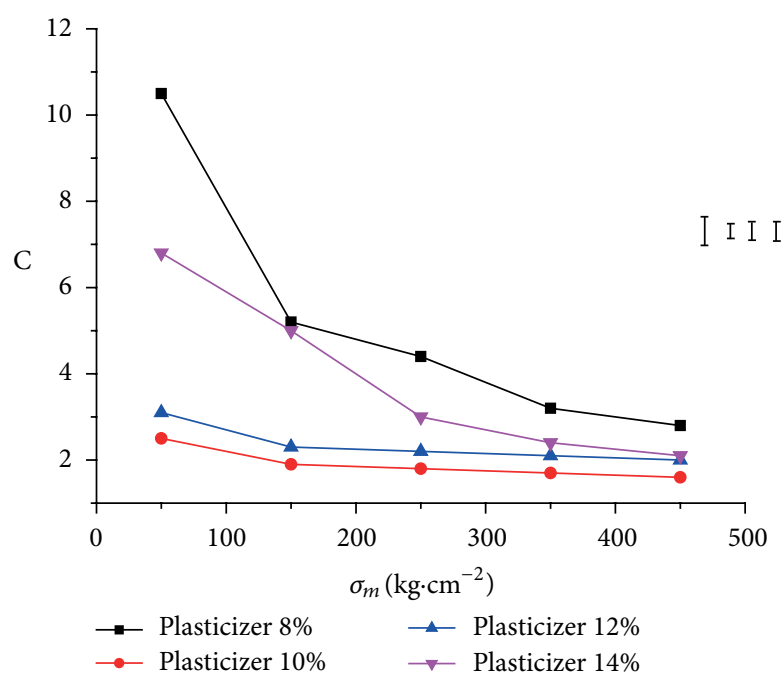

FIGURE 2: Impact of plasticizer content on the cushion coefficientmaximum stress $\left(\mathrm{C}-\sigma_{m}\right)$ curve. Bars indicate mean \pm average standard deviation.

between fiber and starch, which obstructs the water's bridgeconnection [14]. Therefore, considering the cushion performance and processing property, the optimal plasticizer content is $12 \%$. This is consistent with the plasticizer content above determined by the orthogonal experiment, and the cushion performance is better.

\subsubsection{Impact of the Mass Ratio of Fiber to Starch on Cushion} Performance. The mass ratio of fiber to starch is also an important factor to influence the cushion performance. Figure 3 shows the relationship between different mass ratios of fiber to starch and the stress. Experiment conditions were as follows: plasticizer content of $12 \%$, active agent content of $0.3 \%$, and foaming agent content of $0.1 \%$.

As shown in Figure 3, under the same stress condition, the different mass ratios of fiber to starch had a significant impact on the cushion coefficient of the material. When the mass ratio was $1: 4$ or $2: 5$, the cushion coefficient would be smaller, indicating better cushion performance. With the mass ratio increasing, the cushion coefficient decreased first and then increased. The main reasons are as follows. The main component of plant fiber is cellulose consisted of glucose macromolecular chains. The polyhydroxy characteristic of cellulose results in strong hydrogen bonds interaction between cellulose molecules, including intermolecular hydrogen bonds and intramolecular hydrogen bonds. With fiber content increasing, the fiber continuously embeds the starch matrix. Not only the intermolecular hydrogen bonds between the cellulose molecules are formed but also the intramolecular hydrogen bonds between cellulose molecules and starch molecules are formed, as well as the intramolecular hydrogen bonds between the starch molecules and plasticizer molecules. Therefore, a kind of relatively dense mesh structure is formed.

By the infrared spectrum, the wave number of hydroxyl groups' infrared absorption peak decreases first and then

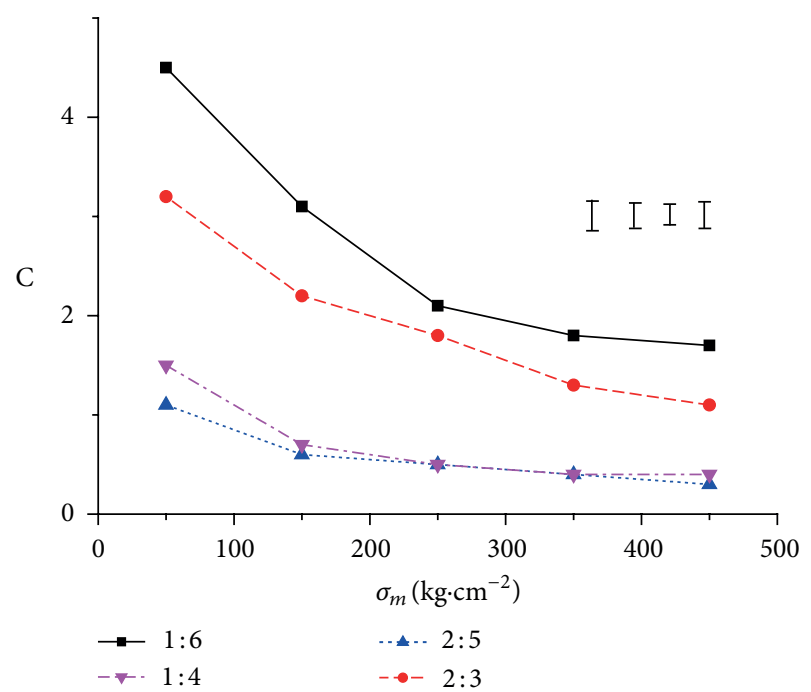

FIgURE 3: Cushion coefficient-maximum stress $\left(\mathrm{C}-\sigma_{m}\right)$ curves of different mass ratios of fiber to starch. Bars indicate mean \pm average standard deviation.

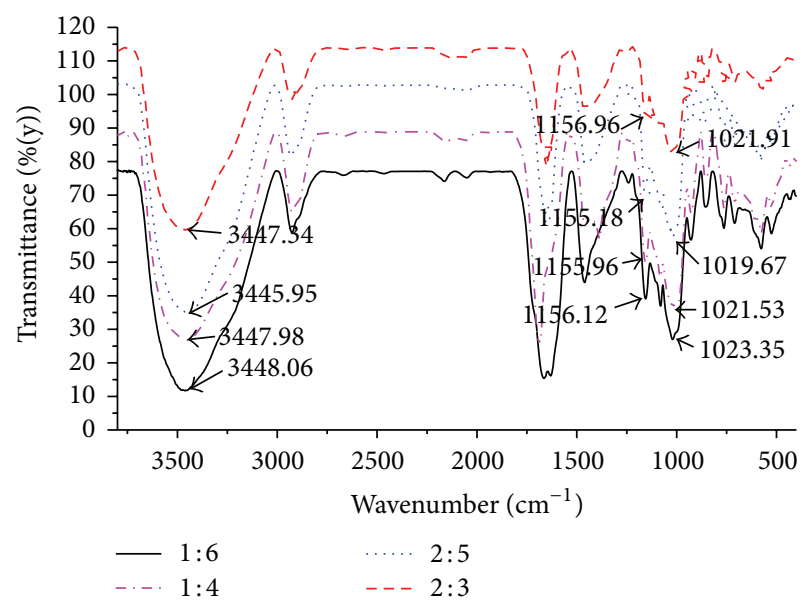

FIGURE 4: Infrared spectra of different ratios of fiber to starch.

increases with the ratio of fiber to starch increasing. As shown in Figure 4, the characteristic of intermolecular hydrogen bonding through hydroxyl groups $(-\mathrm{OH})$ is obvious. The number of hydroxyl groups between fiber and starch has a peak value, indicating that too much fiber or starch is not conducive to form hydrogen bonds. The schematic of water's bridge-connection is shown in Figure 5. In the foaming molding process, the exposed groups of fiber macromolecular chain attract mutually to construct a kind of threedimensional network structure by the form of hydrogen bonds. At the same time, owing to the irregularity of fiber macromolecules, smaller starch molecules also take part in the formation of hydrogen bonds. This kind of new hydrogen bonds plays a cohesive action to promote the fiber's overlapping. When the fiber content is excessive, it is easier for fiber macromolecules to condense clumps because of its hydrophilicity. However, this kind of clumps is more like 


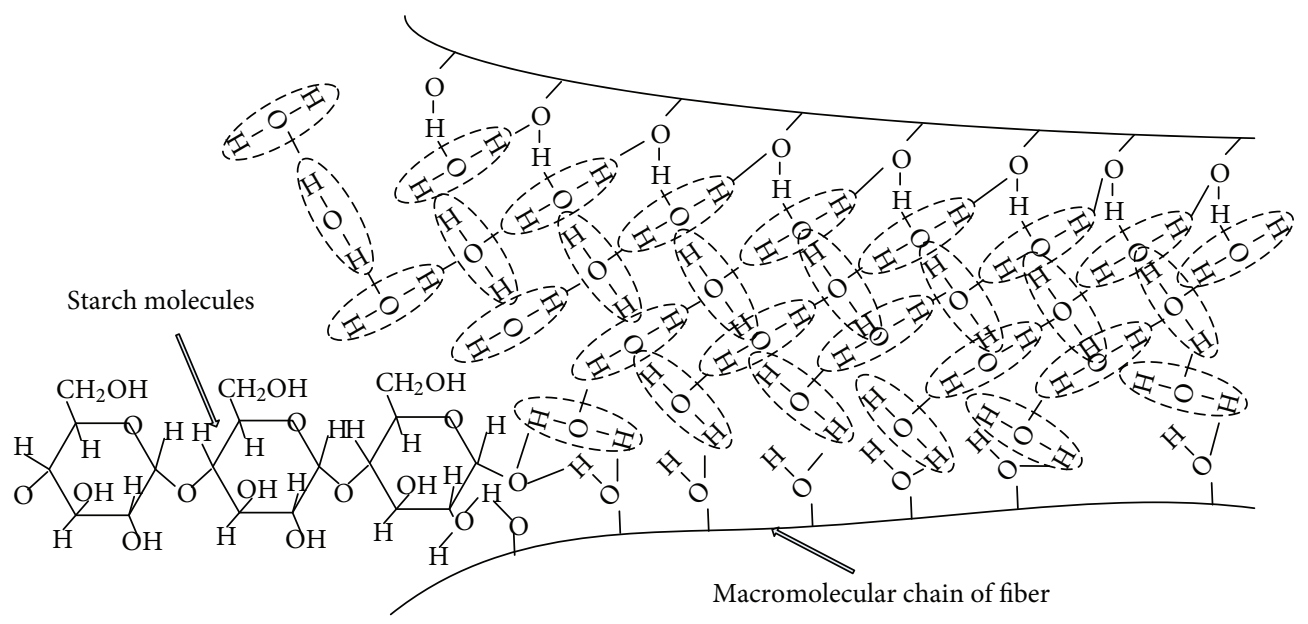

FIGURE 5: Schematic of water's bridge-connection.

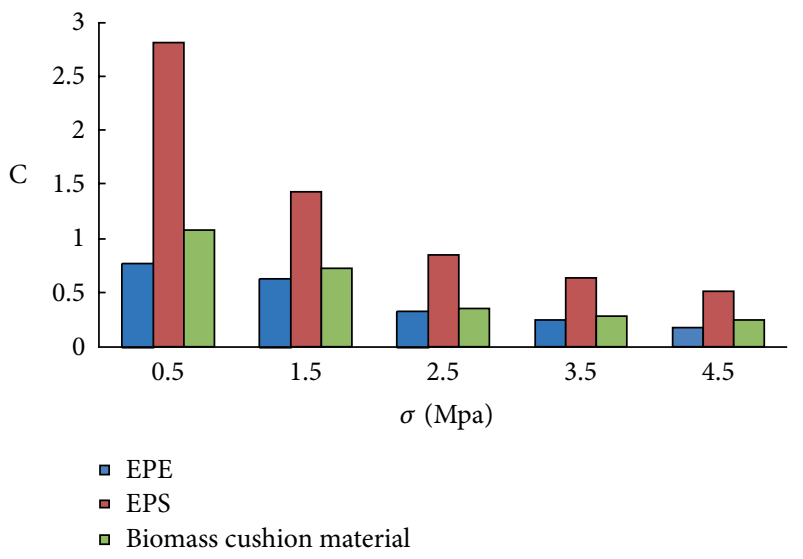

FIGURE 6: Relationship between cushion coefficients and stresses of different packaging materials.

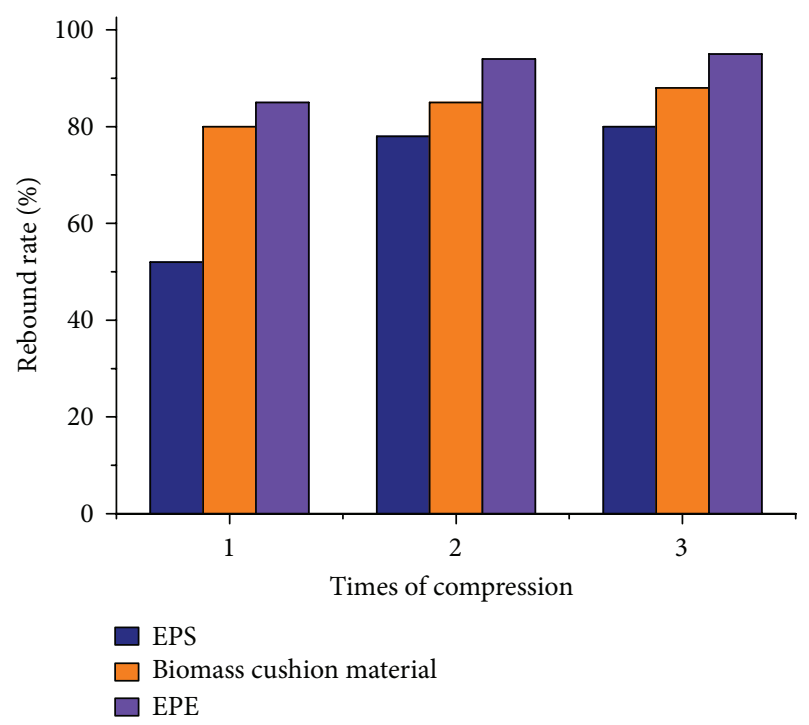

FIGURE 7: Compression rebound ratio for three times. macroscopic appearance. In fact, when lacking of the cohesive action by starch, fiber macromolecules cannot construct the completed network structure by the form of hydrogen bonds, but local overlapping structure is formed. This kind of overlapping decreases the cohesiveness of the material matrix, so that it is hard to form ideal micropore structure. So when the fiber content is excessive, the cushion performance of the biomass material decreases. Similarly, when the starch content is excessive, starch molecules between fiber macromolecules hinder the construction of threedimensional network structure. Therefore, considering the cushion performance and processing property, the optimal mass ratio of fiber to starch is $2: 5$. This is consistent with the mass ratio of fiber to starch determined by the orthogonal experiment above, and the cushion performance is better.

\subsection{Comparison of Several Cushion Packaging Materials}

3.3.1. Cushion Performance. The cushion performance of this material is compared with that of EPE and EPS, as shown in Figure 6. The EPE has no national standard to follow, and its apparent density is about $2 \times 10^{-5} \mathrm{~g} / \mathrm{mm}^{3}$. According to QB/T 1649-92, EPS is the Class II foaming packaging materials, of which the apparent density is about $2.58 \times 10^{-5} \mathrm{~g} / \mathrm{mm}^{3}$. As shown in Figure 5, under the same stress condition, the cushion coefficient of the biomass cushion packaging material is smaller than EPS and higher than EPE. Therefore, cushion performance of the material can meet the general packaging requirements. And it is feasible for this material to serve as cushion liner packaging of small electronic products and meet the protection requirements.

3.3.2. Rebound Performance. Compare the rebound performance of this material with that of EPE and EPS. As shown in Figure 7, the rebound ratio of the material is higher than EPS and lower than EPE. Therefore, the rebound performance of the biomass material can meet general packaging requirements. And interspace between products and the packaging 
container can be avoided with the use of this material, so the secondary impact on products is eliminated.

\section{Conclusion}

(1) The orthogonal experiment method is used to study the impact of the mass ratio of fiber to starch, the content of plasticizer, the active agent, and the foaming agent on the compressive strength of biomass cushion packaging material. The results are demonstrated as follows: the influence of various factors on compressive strength follows the sequence plasticizer $>$ the mass ratio of fiber to starch $>$ foaming agent $>$ active agent. When the mass ratio of fiber to starch is $2: 5$ and the plasticizer content is $12 \%$, the foaming agent content is $0.1 \%$, the active agent content is $0.3 \%$, that is, the optimum ingredient, the compressive strength can reach $0.94 \mathrm{MPa}$.

(2) According to the study on the impact of the plasticizer content and the mass ratio of fiber to starch on cushion performance, the cushion coefficient of the material decreases first and then increases with the two factors increasing. When the plasticizer content is $12 \%$ and the mass ratio of fiber to starch is $2: 5$, the smallest cushion coefficient is obtained; that means the best cushion performance of the material.

(3) Through comparison of the cushion performance and the rebound performance of the biomass packaging material with EPS and EPE, it indicates that the biomass cushion packaging material can totally replace the plastic packaging materials such as EPS and EPE.

\section{Conflict of Interests}

The authors declare that there is no conflict of interests regarding the publication of this paper.

\section{Acknowledgments}

This work was financially supported by the Independent Innovation Foundation of Shandong University (2012JC004), the Natural Science Foundation of Shandong Province (ZR2010EM007), the Natural Science Foundation of China (51275278), and Taishan Scholar Program Foundation of Shandong.

\section{References}

[1] M. O. Rutiaga, L. J. Galan, L. H. Morales et al., "Mechanical property and biodegradability of cast films prepared from blends of oppositely charged biopolymers," Journal of Polymers and the Environment, vol. 13, no. 2, pp. 185-191, 2005.

[2] J. Shey, S. H. Imam, G. M. Glenn, and W. J. Orts, "Properties of baked starch foam with natural rubber latex," Industrial Crops and Products, vol. 24, no. 1, pp. 34-40, 2006.

[3] P. Cinelli, E. Chiellini, J. W. Lawton, and S. H. Imam, "Properties of injection molded composites containing corn fiber and poly(vinyl alcohol)," Journal of Polymer Research, vol. 13, no. 2, pp. 107-113, 2006.
[4] C. N. Ludvik, G. M. Glenn, A. P. Klamczynski, and D. F. Wood, "Cellulose fiber/bentonite clay/biodegradable thermoplastic composites," Journal of Polymers and the Environment, vol. 15, no. 4, pp. 251-257, 2007.

[5] J. Shey, S. H. Imam, G. M. Glenn, and W. J. Orts, "Properties of baked starch foam with natural rubber latex," Industrial Crops and Products, vol. 24, no. 1, pp. 34-40, 2006.

[6] P. Cinelli, E. Chiellini, J. W. Lawton, and S. H. Imam, "Foamed articles based on potato starch, corn fibers and poly(vinyl alcohol)," Polymer Degradation and Stability, vol. 91, no. 5, pp. 1147-1155, 2006.

[7] M. A. Sawpan, K. L. Pickering, and A. Fernyhough, "Effect of fibre treatments on interfacial shear strength of hemp fibre reinforced polylactide and unsaturated polyester composites," Composites A, vol. 42, no. 9, pp. 1189-1196, 2011.

[8] B. K. Goriparthi, K. N. S. Suman, and N. M. Rao, "Effect of fiber surface treatments on mechanical and abrasive wear performance of polylactide/ jute composites," Composites A, vol. 43, no. 10, pp. 1800-1808, 2012.

[9] T. Kunanopparat, P. Menut, M.-H. Morel, and S. Guilbert, "Reinforcement of plasticized wheat gluten with natural fibers: from mechanical improvement to deplasticizing effect," Composites A, vol. 39, no. 5, pp. 777-785, 2008.

[10] X. L. Song and J. Wu, "The influence of plasticizer on the performance of natural plant fibre foaming buffering materials," Packaging Engineering, vol. 26, no. 3, pp. 42-44, 2005.

[11] X. J. Jia, J. F. Li, and F. Y. Li, "Biomass biodegradable food boxes and hot temperature field of structural optimization," in Proceedings of the International Conference on Advances in Materials and Manufacturing Processes (ICAMMP '10), Shenzhen, China, 2011.

[12] M. F. Rosa, B.-S. Chiou, E. S. Medeiros et al., "Effect of fiber treatments on tensile and thermal properties of starch/ethylene vinyl alcohol copolymers/coir biocomposites," Bioresource Technology, vol. 100, no. 21, pp. 5196-5202, 2009.

[13] H. N. Lu, X. J. Jia, J. F. Li, F.-Y. Li, X. Wang, and J.-Y. Li, “Thermal analysis and optimization of mould for fully biodegradable dishware," Die \& Mould Industry, vol. 3, pp. 45-49, 2010.

[14] A. F. Guo, "Research on key technologies of fully-degradable packing materials," Tech. Rep., Shandong University, 2011.

[15] G. Liu, "Research on optimization technology for simulation model of biodegradable production line," Tech. Rep., Shandong University, 2010.

[16] G. Anfu, L. Jianfeng, L. Fangyi, and Y. Yong, "Design of the full biodegradable and single-use dishware mold based on automatic ejection technology," in Proceedings of the 16th IEEE International Symposium on Electronics and the Environment (ISEE '08), pp. 456-457, May 2008.

[17] A.-F. Guo, J.-F. Li, F.-Y. Li, and B.-K. Wei, "Study on the biodegradagility of plant fiber and starch dishware," Journal of Functional Materials, vol. 40, no. 11, pp. 1929-1932, 2009.

[18] A. F. Guo, H. Lu, J. Li et al., "Mechanical property simulation and verification of plant fiber and starch dishware," Transactions of the Chinese Society of Agricultural Engineering, vol. 26, no. 7, pp. 91-95, 2010.

[19] G. Li, F. Y. Li, K. K. Guan, P. Liu, Y. Lv, and J. F. Li, "Preparation and properties of biomass cushion packaging material," Journal of Function Materials, vol. 2, no. 13, pp. 79-85, 2013.

[20] M. X. Zheng, L. Q. Li, M. Y. Zheng et al., "Effect of Alkali pretreatment on cellulosic structural changes of corn stover," Environmental Science \& Technology, vol. 35, no. 26, pp. 27-31, 2012. 

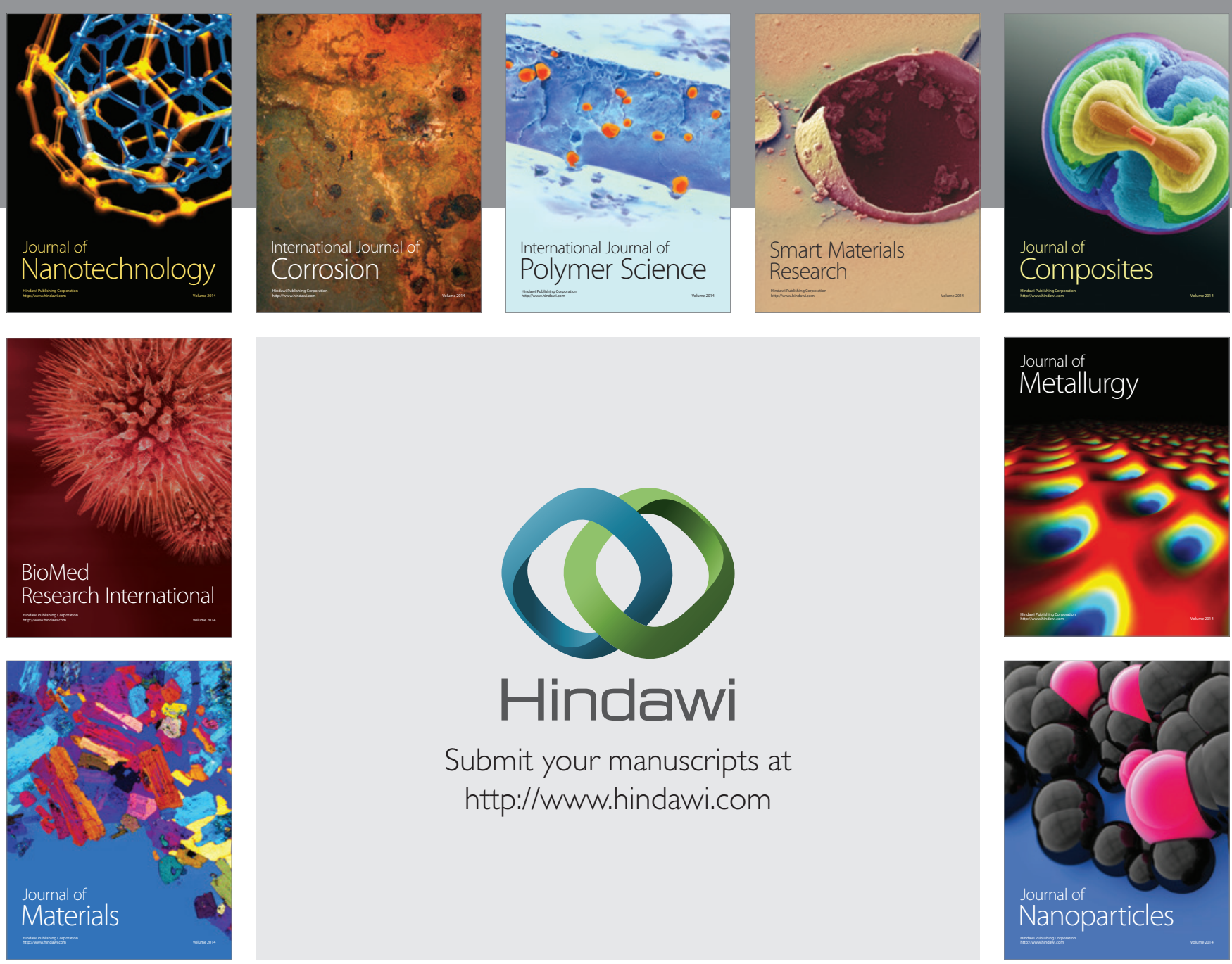

Submit your manuscripts at http://www.hindawi.com
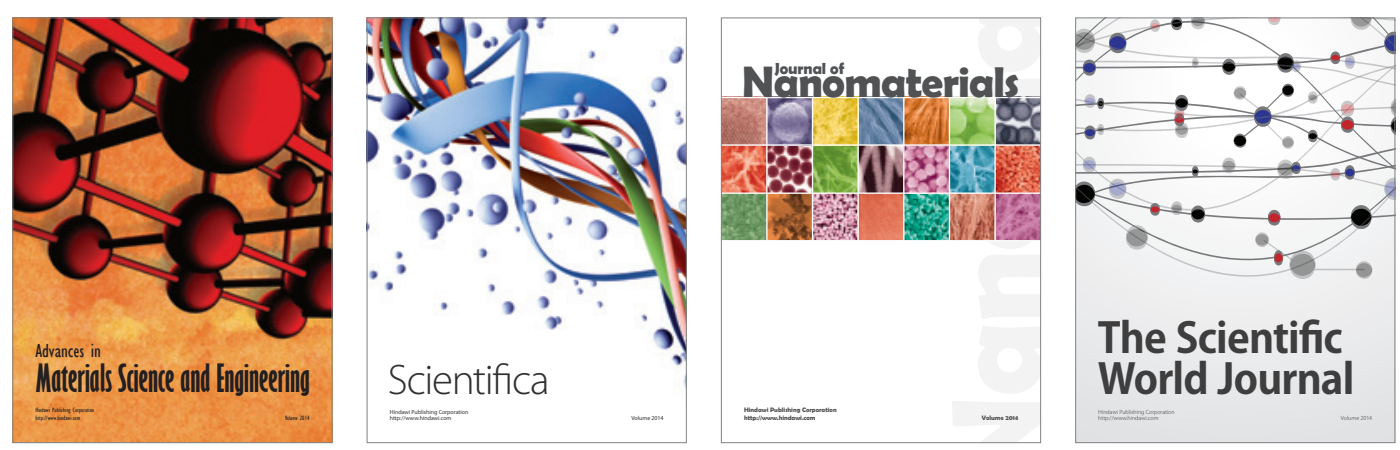

\section{The Scientific World Journal}
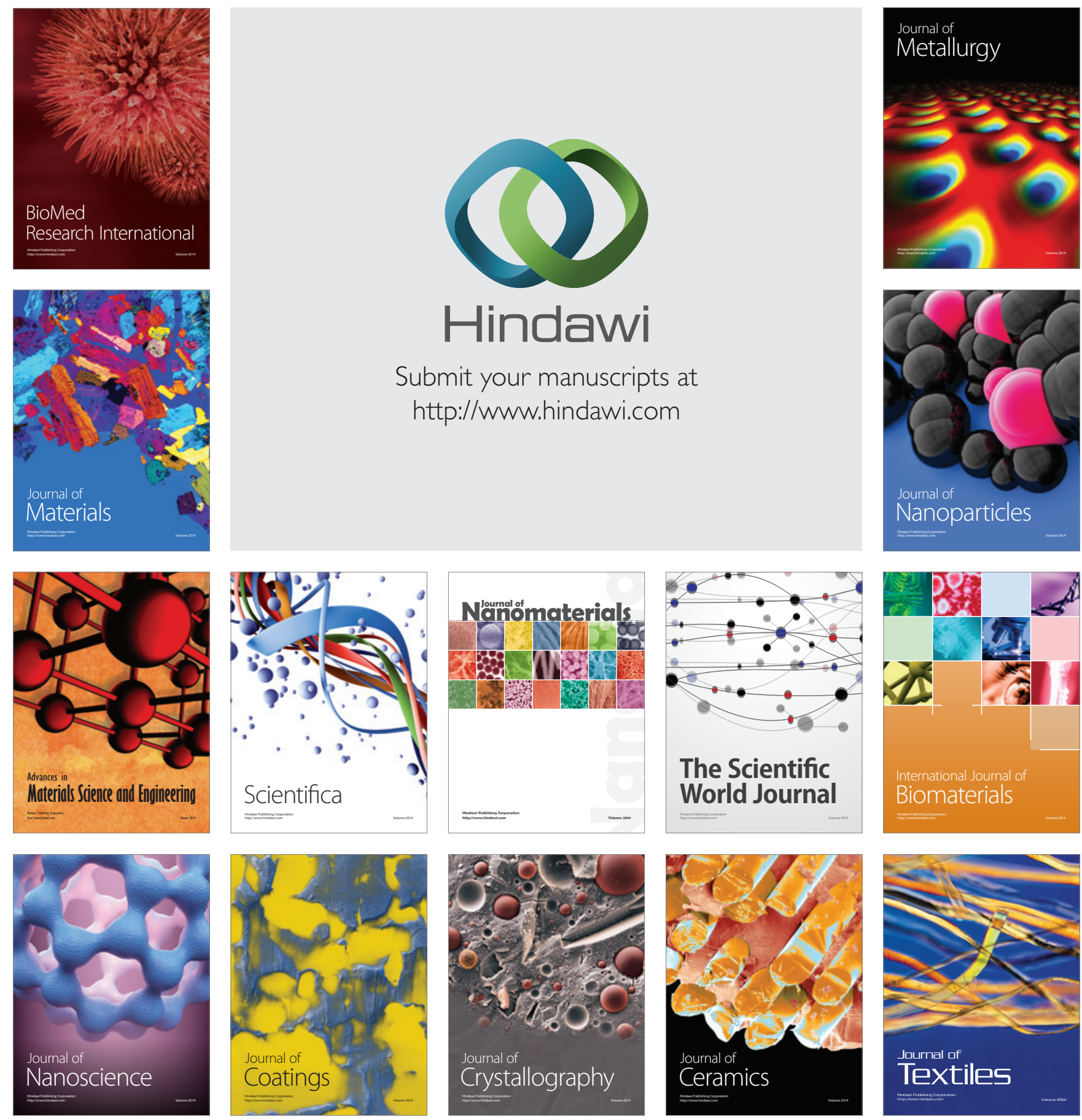